\title{
Impact of acetabular reaming depth on reconstruction of rotation center in primary total hip arthroplasty
}

\author{
Pu Shao ${ }^{1}$, Zhizhou Li ${ }^{1}$, Modi Yang ${ }^{1}$, Yuzhuo Wang ${ }^{3}$, Te Liu², Yuhui Yang ${ }^{1}$, Lian Duan', Jinlan Jiang ${ }^{2 *}$ and
} Jianlin Zuo ${ }^{1 *}$

\begin{abstract}
Purpose: To study the impact of acetabular reaming depth on reconstruction of rotation center $(\mathrm{RC})$ in unilateral primary total hip arthroplasty (UPTHA) and guide individualized preoperative design.

Methods: 200 postoperative standard bilateral hip anteroposterior radiographs after UPTHA were included, which were collected from January, 2013 to June, 2017 in our hospital. Osteonecrosis of femoral head was the only diagnosis in this cohort. The parameters were measured on the anteropoterior radiographs by using RadiAnt DICOM viewer.

Results: The average of the thickness of the teardrop is about $6.13 \pm 1.42 \mathrm{~mm}$. The parameter a (the difference value of the distance of bilateral RC and midline) was positively correlated with the parameter e (the acetabular reaming depth), and the Pearson correlation coefficient was 0.49 when $P=0.05$. Furthermore, the value of parameter (e) was $8.25 \mathrm{~mm}$ when a2 (the distance from the center of the prosthesis femoral head to the vertical line across the midpoint of pubic symphysis) equaled a1 (the distance from RC of the healthy femoral head to the vertical line across the midpoint of pubic symphysis).

Conclusions: The reaming depth of the acetabulum could influence the reconstruction of RC during UPTHA. When the medial margin of the cup was placed about $2 \mathrm{~mm}$ to the lateral border of the ipsilateral teardrop (the bottom of the ovum), the rotation center would be accurately restored.
\end{abstract}

Keywords: Total hip arthroplasty, Rotation center, Acetabulum, Reaming depth, Teardrop

\section{Introduction}

It is now acknowledged that total hip arthroplasty (THA) is one of the most successful procedures in the field of orthopeadics, which is widely applied to the treatment of many hip joint diseases resulted in dysfunction of hip joint [1]. Maximization of the prosthesis life is the most significant issue for orthopedic surgeons [2].

Among the influencing factors for successful restoration of joint, accurate biomechanical reconstruction of the femur and acetabulum is essential for satisfactory function achievement [3-5]. In order to reduce the

\footnotetext{
*Correspondence: jiangj12003@hotmail.com; jlzuojianlin@163.com

${ }^{2}$ Scientific Research Centre of China-Japan Union Hospital of Jilin University,

126 xiantai street, Changchun 130033, People's Republic of China

${ }^{1}$ Department of Orthopedics, China-Japan Union Hospital of Jilin University,

126 xiantai street, Changchun 130033, People's Republic of China
}

Full list of author information is available at the end of the article occurrence of many postoperative complications it is necessary to Reproduce, as close as possible, normal or near-normal mechanics. One of the most important steps in achieving this is to transfer the hip joint $\mathrm{RC}$ into the true acetabulum [6-8]. The native acetabulum is subhemispherical in most cases, but the acetabular components used for THA are hemispherical, which inadvertently leads to displacement of $\mathrm{RC}$ when the acetabular component is fully implanted $[9,10]$. Hence, it is very vital for hip replacement to reconstruct $\mathrm{RC}$ of femoral head prosthesis in the anatomical position of the acetabulum side. Moreover, the RC of hip is also pivotal for recovery of muscle function $[4,11-15]$, joint stability [16-19], and hip prostheses longevity [20-23]. If $\mathrm{RC}$ of femoral head prosthesis is not placed at the anatomical position, it may also lead to patients with

(c) The Author(s). 2018 Open Access This article is distributed under the terms of the Creative Commons Attribution 4.0 International License (http://creativecommons.org/licenses/by/4.0/), which permits unrestricted use, distribution, and reproduction in any medium, provided you give appropriate credit to the original author(s) and the source, provide a link to the Creative Commons license, and indicate if changes were made. The Creative Commons Public Domain Dedication waiver (http://creativecommons.org/publicdomain/zero/1.0/) applies to the data made available in this article, unless otherwise stated. 
unequal limb length, unequal femoral eccentricity, etc. Over time it will cause the muscles to lose their original function, the joints are unstable, and eventually the loosening of the prosthesis.

So, the depth of acetabular prosthesis implantation becomes a main factor infecting the position of RC. Despite advances in surgery technique, the landmark of acetabular reaming depth for anatomic restoration of RC remains uncertain. In this paper, standard bilateral hip anteroposterior radiographs after unilateral primary THA (UPTHA) were used to clarify the accurate acetabular reaming depth for anatomically reconstructing RC of hip joint during THA.

\section{Methods}

\section{General information}

In this study, 200 standard bilateral hip anteroposterior radiographs after UPTHA were collected from January 2013 to June 2017 in our hospital. As shown in Table 1, there were 116 males and 84 females in all subjects. Among them, there were 97 patients on the left side and 103 patients on the right side. The average age was 58.1 years (from 24 to 80 years). The inclusion criterions applied was as follow: Patients with unilateral osteonecrosis of femoral head while the contralateral hip joint was normal. The exclusion criterions used were as follow: 1 Patients with unilateral or bilateral acetabular or femoral developmental dysplasia; 2. Patients with unilateral or bilateral hip joint fracture history.

\section{$\mathrm{X}$-ray and measurements}

The standard bilateral hip anteroposterior radiographs were gathered after UPTHA. The criteria for taking the radiographs were as follow: The patients were supine on the photography table, with median sagittal plane coincided with the cassette midline, the lower limbs were fully extended, and were placed in internal rotation about $15^{\circ}$. The X-ray filter was used. The tube distance was $100 \mathrm{~cm}$. The center of the beam was focused on the point $3 \mathrm{~cm}$ below the pubic symphysis midpoint, and the beam was vertically injected into the cassette. The position was controlled so as to make the bilateral obturator

Table 1 General information of the 200 patients

\begin{tabular}{llll}
\hline Item & Classification & Number & Percentage \\
\hline Sexuality & Male & 116 & $58 \%$ \\
& Female & 84 & $42 \%$ \\
Age & $<40$ & 12 & $6 \%$ \\
& $40-60$ & 100 & $50 \%$ \\
\multirow{3}{*}{ Operation Side } & $>60$ & 88 & $44 \%$ \\
& Left & 97 & $48.5 \%$ \\
& Right & 103 & $51.5 \%$ \\
\hline
\end{tabular}

foramens isometrical and the tip of coccyx locating at the level and in the center of the pubic symphysis. The longitudinal axes of bilateral femurs were parallel to each other and to the longitudinal central axis of the pelvis.

As shown in Fig. 1, RadiAnt DICOM viewer (version 1.9.16, 32-bit, developed by Medixant Company in Poland in 2009) was applied to measure the following parameters on the above mentioned postoperative standard bilateral hip anteroposterior radiographs. First a transverse line passing through the lower edges of bilateral teardrops were drawn. Then the following measurements were made: a1. The distance from $\mathrm{RC}$ of the healthy femoral head to the vertical line across the midpoint of pubic symphysis; a2. The distance from the center of the prosthesis femoral head to the vertical line across the midpoint of pubic symphysis; b1. The diameter of the healthy femoral head; b2. The diameter of the femoral head prosthesis; c. The thickness of the teardrop on the contralateral side; $d$. The distance between the vertical lines tangent to the medial margins of the femoral head prosthesis and the acetabular prosthesis; e. The distance between the vertical lines tangent to the medial margins of the acetabular prosthesis and the medial border of the ipsilateral teardrop, which was defined as the horizontal reaming depth.

\section{Correction and standardization of the measurements}

After the measurement of the above parameters, the value correction was done. Parameter b2, the diameter of the femoral head prosthesis, was a known data, which could be retrieved from the patient chart. So, the ratio of the true value and the measured value of the femoral head prosthesis could be obtained in each case, which was named as correction coefficient. By multiplying each patient's data by the corresponding correction coefficient, the true value of each parameter could be obtained in each case. Standardization of the measured data was subsequently carried out in order to make the results comparable among the 200 patients with different heights and weights. After the measurements and corrections, the ratio of the diameter of the largest to the diameter of every other femoral head, called standardized coefficient, was obtained. Then, by multiplying the true value of each parameter with the standardized coefficient the standard value of each parameter was acquired.

\section{Statistical analysis}

SPSS 22.0 was applied for statistical analysis. Pearson correlation analysis was used to analyze the correlation between the parameter (a) $(\mathrm{a}=\mathrm{a} 2-\mathrm{a} 1)$ and the parameter (e), and the pattern of relevant scatter plot was drawn. The illustration of relationship of parameter (e) and parameter (a) was further progressed by regression analysis. 


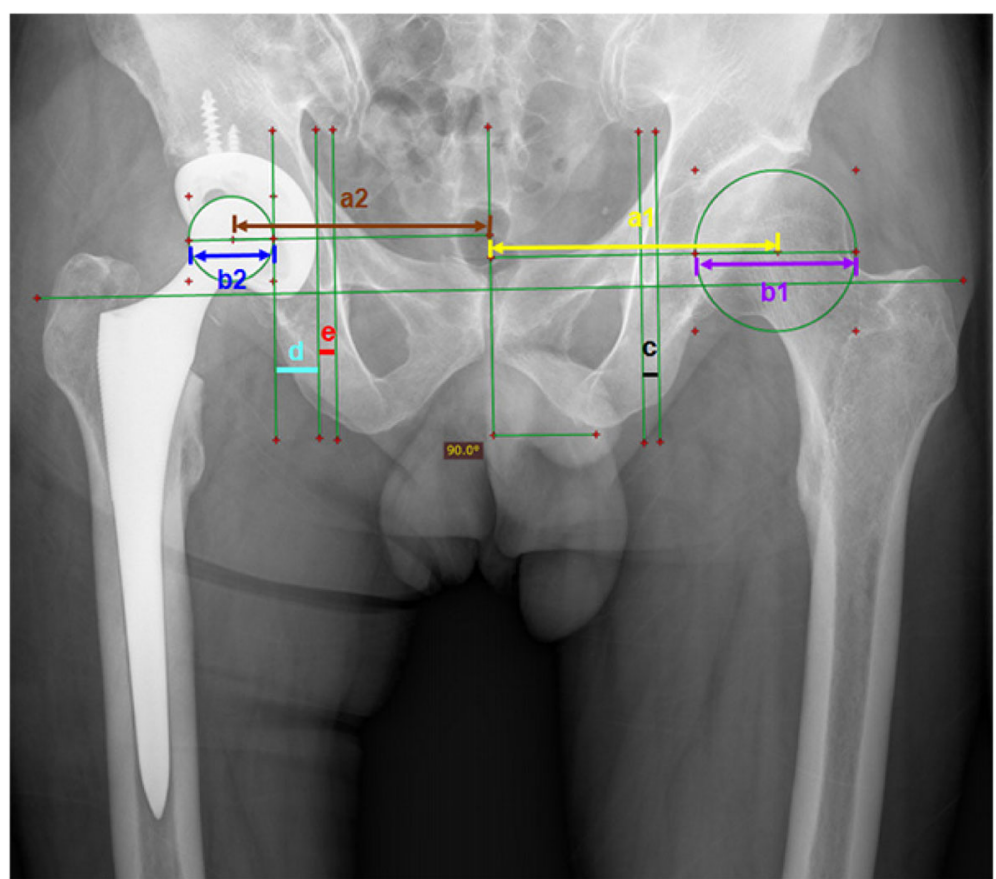

Fig. 1 Postoperative measurement: the distance between the center of rotation of the healthy femoral head to the vertical line across the midpoint of pubic symphysis (a1); the distance between the center of the prosthesis femoral head to the vertical line across the midpoint of pubic symphysis (a2); the diameter of the healthy femoral head (b1); the diameter of the prosthesis femoral head (b2); the thickness of the teardrop(c); the distance between the medial edge of prosthesis femoral head and the medial edge of the acetabular cup (d); the distance between the medial edge of the acetabular cup and the medial edge of teardrop (e)

\section{Results}

The diameter of the largest femoral head on the healthy side was $55.5 \mathrm{~mm}$. After the Pearson correlation analysis is performed for parameter a (the difference value of the distance of bilateral RC and midline) and parameter $\mathrm{e}$ (the distance between the vertical lines tangent to the medial margins of the acetabular prosthesis and the medial border of the ipsilateral teardrop), it can be concluded that the parameter (a) was positively correlated with the parameter (e), and the Pearson correlation coefficient was 0.49 , when $P=0.05$. That is to say the parameter (e) is increased with an increase in the parameter (a). As shown in Fig. 2, the pattern of the relevant scatter plot was suggested that the parameter (a) was positively correlated with the parameter (e). In addition, the regression analysis was further shown that the coefficients of the independent variables passed the statistical test at a confidence level of 0.05 . The regression equation was finally established as follow:

$$
\mathrm{e}=8.25+0.17 \times(\mathrm{a} 2-\mathrm{a} 1)
$$

Hence, when the RC of the operated hip was restored anatomically in the horizontal direction, that is (a) $=0$, the distance between the medial tangent line of the acetabular prosthesis and that of the lateral border of the ipsilateral teardrop, that is e, equaled $8.25 \mathrm{~mm}$. This result was got at the condition that the diameter of the femoral head was $55.5 \mathrm{~mm}$. Because of the medial margin of the teardrop cannot be seen during operation, we measured the thickness of teardrop, which is $6.13 \mathrm{~mm} \pm$ $1.42 \mathrm{~mm}$. Then we drew the conclusion that when the medial margin of the cup was about $2 \mathrm{~mm}(8.25 \mathrm{~mm}$ minus $6.13 \mathrm{~mm}$ equals about $2 \mathrm{~mm}$ ) lateral to the lateral margin of the ipsilateral teardrop the rotation center of the operated hip would be anatomically restored.

\section{Discussion}

In all precedures of preoperative plan for THA, the anatomical reconstruction of acetabular RC is a very important target [24, 25]. The position of RC of the operated hip was the most important factor for the stress and function of the soft tissue around the hip joint. And in the anatomical reconstruction of the horizontal of the $\mathrm{RC}$, the horizontal reaming depth of the acetabulum plays a very important role. The ideal position of acetabular prosthesis mainly depended on the femoral head size and the neck diameter. For the long-term service life of the prosthesis, the acetabulum should be placed in its anatomical position to normally inclusion of the acetabular prosthesis and restore the 


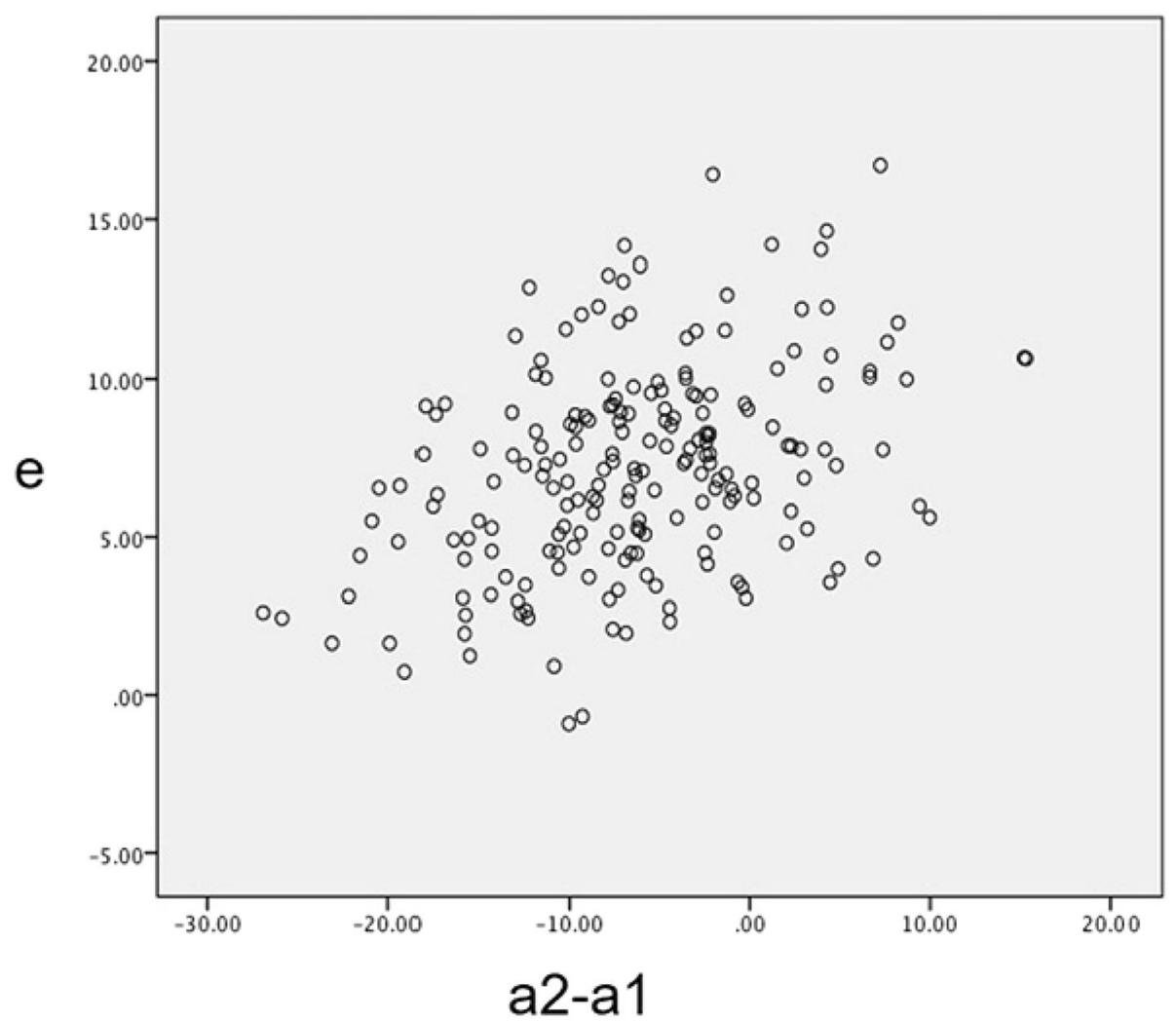

Fig. 2 The pattern of linear correlation scatter plot of the reaming depth (e) and the difference value of the distance of bilateral RC and midline $(\mathrm{a} 2-\mathrm{a} 1)$

length of the limb [26]. In the existing literature reports, no one has studied the goal of the ideal depth of acetabular reaming. The acetabular reaming depth affects the position of the RC in horizon direction. If the reaming depth is too deep or too shallow, it may affect the survivorship of the prosthesis by changing the force torque of the reconstructed hip. There is no uniform standard for acetabular prosthesis depth in THA, so far. In this paper, standard bilateral hip anteroposterior radiographs after unilateral primary THA (UPTHA) were used to clarify the accurate acetabular reaming depth for anatomically reconstructing $\mathrm{RC}$ of hip joint during THA. And it will have a great potential in individualized preoperative plan of THA.

In this paper, the distance (a2) between the RC of operated and midline can be divided into three parts: the radius of femoral head component (half of b2), the thickness of acetabular cup (d) (including metal acetabular cup and high molecular polyethylene liner), and the reaming depth (e) (the distance between the medial tangent line of the acetabular component and that of the medial border of the ipsilateral teardrop) in the postoperative anteroposterior radiographs of THA. Because the radius of femoral head component and the thickness of acetabular component were two constant values, after the data correction and standardization, the reaming depth (e) became the only one variable affecting the reconstruction of RC. In this paper, we did correction to exclude influence of the magnification of the radiographs by calculating the ratio of the true value of the femoral head prosthesis (was a known data, which could be retrieved from the patient chart.) and the measured value of the femoral head prosthesis. Then we performed standardization to exclude influence of bone size on the measurements. We used diameter of the largest healthy femoral head $(55.5 \mathrm{~mm})$ in the 200 patients as the standard, the measurements of other patients were then multiplied by the ratio of the diameter of the corresponding femoral head to that of the largest femoral head $(55.5 \mathrm{~mm})$. By balancing different sizes of bone from this cohort of patients, we could analyze if there were linear correlations between the parameters. According to statistical analysis, it was suggested that the reaming depth (e) in horizon direction would be 8.25 $\mathrm{mm}$ under the condition that the diameter of healthy femoral head was $55.5 \mathrm{~mm}$ and $\mathrm{RC}$ was restored anatomically ( $\mathrm{a} 1=\mathrm{a} 2)$.

It is well known that the medial margin of teardrops is visible in the radiographs imaging but invisible in the surgery procedure. But the lateral border of the teardrop 
is visible in the surgery procedure. It is the bottom of the ovum. Therefore, we further measured the thickness of the teardrop(c), so as to obtain the best distance between the vertical lines tangent to the medial margins of the acetabular cup and the lateral border of the teardrop. Then we drew the conclusion that when the medial margin of the cup was about $2 \mathrm{~mm}(8.25 \mathrm{~mm}$ minus 6.13 $\mathrm{mm}$ equals about $2 \mathrm{~mm}$ ) lateral to the lateral margin of the ipsilateral teardrop the rotation center of the operated hip would be anatomically restored. Consequently, in this work, a new standardized method of restoration of $\mathrm{RC}$ was provided, and it will have a great potential in individualized preoperative plan of THA.

The limitation of this study is that the thickness of the cup prosthesis varies a little bit among different brands, and the thickness of the linear changes with the size of the cup. And we did not record height and weight of the patients in this cohort. But the influences of the former two factors were already minimized by standardization of the data.

\section{Conclusion}

We found that the reaming depth of the acetabulum influence the reconstruction of $\mathrm{RC}$ during THA. In the procedures of individualized preoperative plan, the distance between the medial tangent line of the acetabular prosthesis and that of the medial border of the ipsilateral teardrop, $\mathrm{e}=8.25 \mathrm{~mm}$, when the diameter of the healthy femoral head was $55.5 \mathrm{~mm}$. Because of the average thickness of the normal teardrop(c) is $6.13 \pm 1.42 \mathrm{~mm}$, we can conclude that when the medial margin of the cup was placed about $2 \mathrm{~mm}$ to the lateral border of the ipsilateral teardrop (the bottom of the ovum), the rotation center would be accurately restored.

\section{Abbreviations}

RC: Rotation center; THA: Total hip arthroplasty; UPTHA: Unilateral primary total hip arthroplasty

\section{Acknowledgements}

None

\section{Funding}

"This work was supported and funded by the National Nature Science Foundation of China (No. 81701811)."

\section{Availability of data and materials}

I do not wish to share my data because they involve the privacy of patients in our hospital. But the datasets used and/or analyzed during the current study are available from the corresponding author on reasonable request.

\section{Authors' contributions}

$P S, Z L, M Y$ and $Y W$ completed the data measurement. $T L, Y Y$ and $L D$ completed the statistical analysis. PS completed the article writing. At last JZ and JJ completed the article changes. After the review comments were sent back, JJ completed the revision of the manuscript according to the reviewer's request. All authors read and approved the final manuscript.

\section{Ethics approval and consent to participate}

This study was approved by the local institutional review board of ChinaJapan Union Hospital of Jilin University (No. 201601007). Written informed consent (including patients' details) was obtained from all participants. This study was conducted in accordance to the Declaration of Helsinki.

\section{Consent for publication}

Not applicable.

\section{Competing interests}

The authors declare that they have no competing interests.

\section{Publisher's Note}

Springer Nature remains neutral with regard to jurisdictional claims in published maps and institutional affiliations.

\section{Author details}

'Department of Orthopedics, China-Japan Union Hospital of Jilin University, 126 xiantai street, Changchun 130033, People's Republic of China. ${ }^{2}$ Scientific Research Centre of China-Japan Union Hospital of Jilin University, 126 xiantai street, Changchun 130033, People's Republic of China. ${ }^{3}$ Department of Orthodontics, School and Hospital of Stomatology, Jilin University,

Changchun 130021, People's Republic of China.

Received: 20 March 2018 Accepted: 7 November 2018

Published online: 30 November 2018

\section{References}

1. Bhaskar D, Rajpura A, Board T. Current concepts in Acetabular positioning in Total hip Arthroplasty. Indian J Orthop. 2017;51(4):386-96.

2. Bouffard V, Begon M, Champagne A, Farhadnia P, Vendittoli PA, Lavigne M, Prince F. Hip joint center localisation: a biomechanical application to hip arthroplasty population. World J Orthop. 2012;3(8):131-6.

3. Kiyama T, Naito M, Shinoda T, Maeyama A. Hip abductor strengths after total hip arthroplasty via the lateral and posterolateral approaches. J Arthroplast. 2010:25(1):76-80.

4. Asayama I, Chamnongkich S, Simpson KJ, Kinsey TL, Mahoney OM. Reconstructed hip joint position and abductor muscle strength after total hip arthroplasty. J Arthroplast. 2005;20(4):414-20.

5. Erceg M. The influence of femoral head shift on hip biomechanics: additional parameters accounted. Int Orthop. 2009;33(1):95-100.

6. Kilicoglu OI, Turker M, Akgul T, Yazicioglu O. Cementless total hip arthroplasty with modified oblique femoral shortening osteotomy in Crowe type IV congenital hip dislocation. J Arthroplast. 2013;28(1):117-25.

7. Erdemli B, Yilmaz C, Atalar H, Guzel B, Cetin I. Total hip arthroplasty in developmental high dislocation of the hip. J Arthroplast. 2005;20(8):1021-8.

8. Muratli KS, Karatosun V, Uzun B, Celik S. Subtrochanteric shortening in total hip arthroplasty: biomechanical comparison of four techniques. J Arthroplast. 2014;29(4):836-42.

9. Meermans G, Doorn JV, Kats JJ. Restoration of the Centre of rotation in primary total hip arthroplasty: the influence of acetabular floor depth and reaming technique. Bone Joint J. 2016;98-B(12):1597-603.

10. Kohnlein W, Ganz R, Impellizzeri FM, Leunig M. Acetabular morphology: implications for joint-preserving surgery. Clin Orthop Relat Res. 2009;467(3): 682-91.

11. Delp SL, Maloney W. Effects of hip center location on the momentgenerating capacity of the muscles. J Biomech. 1993;26(4-5):485-99.

12. Doehring TC, Rubash HE, Dore DE. Micromotion measurements with hip center and modular neck length alterations. Clin Orthop Relat Res. 1999; (362):230-9.

13. Doehring TC, Rubash HE, Shelley FJ, Schwendeman $\sqcup$, Donaldson TK, Navalgund YA. Effect of superior and superolateral relocations of the hip center on hip joint forces An experimental and analytical analysis. J Arthroplasty. 1996;11(6):693-703.

14. Johnston RC, Brand RA, Crowninshield RD. Reconstruction of the hip. A mathematical approach to determine optimum geometric relationships. J Bone J Surg Am Vol. 1979;61(5):639-52

15. McGrory BJ, Morrey BF, Cahalan TD, An KN, Cabanela ME. Effect of femora offset on range of motion and abductor muscle strength after total hip arthroplasty. J Bone Joint Surg British Vol. 1995;77(6):865-9. 
16. Kurtz WB, Ecker TM, Reichmann WM, Murphy SB. Factors affecting bony impingement in hip arthroplasty. J Arthroplast. 2010;25(4):624 634 e621-622.

17. Garcia-Rey E, Garcia-Cimbrelo E. Abductor biomechanics clinically impact the Total hip Arthroplasty dislocation rate: a prospective long-term study. J Arthroplast. 2016;31(2):484-90.

18. Sariali E, Klouche S, Mamoudy P. Investigation into three dimensional hip anatomy in anterior dislocation after THA. Influence of the position of the hip rotation Centre. Clin Biomech. 2012;27(6):562-7.

19. Timperley AJ, Biau D, Chew D, Whitehouse SL. Dislocation after total hip replacement - there is no such thing as a safe zone for socket placement with the posterior approach. Hip Int. 2016;26(2):121-7.

20. Callaghan JJ, Salvati EA, Pellicci PM, Wilson PD Jr, Ranawat CS. Results of revision for mechanical failure after cemented total hip replacement, 1979 to 1982. A two to five-year follow-up. J Bone Joint Surg Am Vol. 1985;67(7): 1074-85.

21. Karachalios T, Hartofilakidis G, Zacharakis N, Tsekoura M. A 12- to 18-year radiographic follow-up study of Charnley low-friction arthroplasty The role of the center of rotation. Clin Orthop Relat Res. 1993;296:140-7.

22. Lachiewicz PF, McCaskill B, Inglis A, Ranawat CS, Rosenstein BD. Total hip arthroplasty in juvenile rheumatoid arthritis. Two to eleven-year results. J Bone Joint Surg Am Vol. 1986;68(4):502-8.

23. Yoder SA, Brand RA, Pedersen DR, O'Gorman TW. Total hip acetabular component position affects component loosening rates. Clin Orthop Relat Res. 1988;(228):79-87.

24. Takamatsu T, Shishido T, Takahashi Y, Masaoka T, Tateiwa T, Kubo K, Endo K, Aoki M, Yamamoto K. Radiographic determination of hip rotation center and femoral offset in Japanese adults: a preliminary investigation toward the preoperative implications in Total hip Arthroplasty. Biomed Res Int. 2015;2015:610763

25. Bjarnason JA, Reikeras O. Changes of center of rotation and femoral offset in total hip arthroplasty. Ann Trans Med. 2015;3(22):355.

26. Kluess D, Martin H, Mittelmeier W, Schmitz KP, Bader R. Influence of femoral head size on impingement, dislocation and stress distribution in total hip replacement. Med Eng Phys. 2007;29(4):465-71.

Ready to submit your research? Choose BMC and benefit from:

- fast, convenient online submission

- thorough peer review by experienced researchers in your field

- rapid publication on acceptance

- support for research data, including large and complex data types

- gold Open Access which fosters wider collaboration and increased citations

- maximum visibility for your research: over $100 \mathrm{M}$ website views per year

At $\mathrm{BMC}$, research is always in progress.

Learn more biomedcentral.com/submissions 